\title{
Supporting the One-Carbon Cycle Restores Ovarian Reserve in Subfertile Women: Absence of Correlation with Urinary Bisphenol A Concentration
}

Erica Silvestris, ${ }^{1,2}$ Marc Cohen, ${ }^{2}$ Dominique Cornet, ${ }^{3}$ Laetitia Jacquesson-Fournols, ${ }^{3}$ Patrice Clement, ${ }^{4}$ Jacques Chouteau, ${ }^{5}$ Marc Schneider, ${ }^{6}$ Thierry Besnard, ${ }^{7}$ and Yves Ménézo ${ }^{4-8, *}$

\begin{abstract}
Environmental endocrine disrupting chemicals (EDCs), including bisphenol A (BPA), induce DNA methylation errors and oxidative stress, and alter fertility. Animal studies have demonstrated that supporting the one-carbon cycle (1-CC) with appropriate dietary supplements can reduce the effects of EDCs. Anti-Mullerian hormone $(\mathrm{AMH})$, a marker of ovarian functionality, has been tested in subfertile female patients, to control this hypothesis in humans. Fifty-five women with a history of 3-7 years of infertility, with at least two assisted reproductive technology (ART) treatment failures, and low serum levels of AMH were enrolled in the study. Before starting any further ART treatment, they were tested for AMH and for follicular count. A urinary control of BPA was proposed. Then a support of the 1-CC, already tested in other clinical studies, was initiated and continued for 4 months. At the end of this period, antral follicle count and serum AMH levels were re-evaluated. The AMH levels before and after treatment were compared using the Wilcoxon test (nonparametric test, non-Gaussian population). Out of the 55 patients, 35 accepted a BPA dosage in the urine. No correlation was found between BPA and serum AMH concentrations. Forty-nine patients followed the full treatment with 1-CC supplements, which resulted in increased AMH levels, independent of initial AMH levels and maternal age (in the range studied), $p=0.0001$. Eight patients spontaneously conceived ongoing pregnancies within 3 months, at the end of the protocol. A support of the 1-CC can partly alleviate metabolic derangements induced by environment, as observed in animal models, and improve endocrine background in women.
\end{abstract}

Keywords: anti-Mullerian hormone (AMH); bisphenol A; infertility; one-carbon cycle (1-CC); ovarian reserve; women

\section{Introduction}

Gametogenesis involves important epigenetic reprogramming associated with DNA and histone methylation. During a specific developmental window, the processes of epigenetic maintenance and then reprogramming are sensitive to pressures caused by environ- mental exposure, with biochemical variations that can alter the health/viability, development, and even the survival of mammalian embryos. ${ }^{1,2}$ The negative impact of abnormal sperm DNA methylation on male fertility and the health of the conceptus has been clearly demonstrated. ${ }^{3-5}$ Controlled ovarian hyperstimulation

\footnotetext{
'Division of Gynecology and Obstetrics, University of Bari "Aldo Moro," Bari, Italy.

${ }^{2}$ Clinique Natecia, Lyon, France.

${ }^{3}$ Clinique de la Muette, Rue Nicolo, Paris, France.

${ }^{4}$ Laboratoire Clément, Paris, France.

${ }^{5}$ Laboratoire Oriade, Grenoble, France.

${ }^{6}$ Clinique Belledone, Saint-Martin-d'Hères, France.

${ }^{7}$ Laboratoire LTB, Narbonne, France.

${ }^{8}$ London Fertility Associates, London, England.
}

*Address correspondence to: Yves Ménézo, PhD, Dr Sci, London Fertility Associates, Harley Street, London, England, E-mail: yves.menezo@gmail.com

(c) Erica Silvestris et al. 2017; Published by Mary Ann Liebert, Inc. This is an Open Access article distributed under the terms of the Creative Commons Attribution License, which permits unrestricted use, distribution, and reproduction in any medium, provided the original work is properly cited. 
or elevated homocysteine levels can alter methylation imprints on oocytes and early embryos. ${ }^{6-8}$

DNA methylation is of major importance in gametogenesis because primordial germ cells undergo a process of DNA demethylation when they enter the developing gonads (imprint erasure). This process is subsequently reversed during prenatal life in males and during postnatal follicle development in females. Correct maintenance of methylation is a prerequisite for preimplantation development. ${ }^{6,9}$

Environmental endocrine disrupting chemicals (EDCs) are widely present in products for "domestic use," and even in cosmetics: they are major factors that can disrupt DNA methylation and, as a consequence, epigenetics. In the rat, plastic derivatives such as bisphenol A (BPA), di(2-ethylhexyl)phthalate (DEHP), and dibutyl phthalate (DBP) induce transgenerational reproductive and metabolic pathologies. ${ }^{10-14}$ EDCs induce anomalies in methylation profiles and regulatory epigenetic mechanisms, and are involved in their transgenerational transmission. DNA/histone methylation anomalies are significantly involved in these pathologies. ${ }^{15}$ The link between EDCs, oxidative stress, and DNA methylation anomalies is now well established: EDCs generate oxidative stress through estrogen receptors and peroxisome proliferator-activated receptors. ${ }^{9}$ BPA has a strong structural similarity with estradiol and diethylstilbestrol.

Human epidemiological studies have recently highlighted a correlation between the presence of BPA, parabens, and other organic pollutants in the urine or serum of women who have difficulty in conceiving and/or suffer early menopause. ${ }^{11,12}$ Similarly, phthalates have been found in the urine of subfertile men. ${ }^{13}$ This is probably linked to the significant relationship between sperm quality and errors in sperm DNA methylation. ${ }^{3,4}$ EDCs have a direct negative effect on rodents' ovaries and testes, and also affect the health of the pups. ${ }^{10-14}$ Experiments in the Avy/a (yellow) mouse have shown that methyl donor supplements (i.e., support of the one-carbon cycle [1-CC]) positively affect gene expression and counteract the hypomethylation effects of BPA. ${ }^{15-17}$ The 1-CC (Fig. 1) is involved in methylation processes, by recycling of homocysteine, and generation of endogenous antioxidants such as glutathione and hypotaurine, as well as CoQ10, capable of modulating epigenetic settings ${ }^{18}$ in association with vitamins B2 and B3. Recent preliminary clinical studies ${ }^{19-21}$ confirm the validity of supporting the 1-CC in subfertile couples, to counteract the dual negative effects of the environment. Treating female patients who had failed previous in vitro fertilization

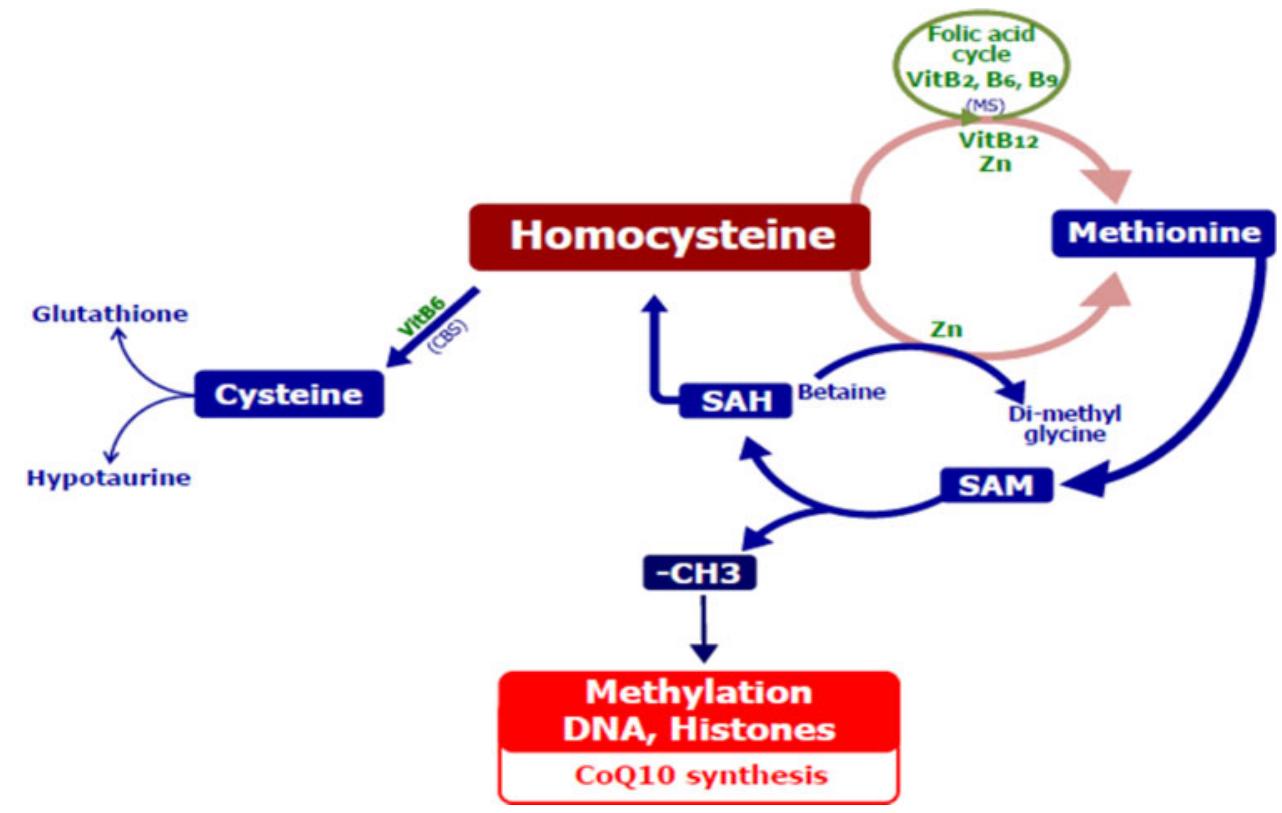

FIG. 1. The one-carbon cycle: contribution to methylation process and genesis of major antioxidant molecules. 
treatments with 1-CC supplements before entering new ART protocols has resulted in surprisingly spontaneous pregnancy rates. ${ }^{9,19-21}$

Anti-Mullerian hormone (AMH) has a pivotal role in female fertility, as a major factor in both folliculogenesis and ovarian cell steroidogenesis. It is secreted by early preantral and antral follicles, and circulating AMH levels correlate directly with functional ovarian reserve. AMH is thus regarded a biomarker of ovarian function, and its measurement in clinical practice is apparently helpful in identifying patients at risk of accelerated decline in ovarian reserve. Patients with low AMH $(0.2-1 \eta \mathrm{g} / \mathrm{mL})$, independent of their age, are considered having very poor chances of pregnancy, even when helped by Assisted Reproductive Technologies. $^{22}$ This study addressed the following question: since negative effects of environmental endocrine disruptors can be reversed by supporting methylation processes in an animal model, is it possible to observe a similar effect in humans? We assayed urinary BPA in a group of female volunteers with very low serum AMH levels to determine whether a correlation could be found. We then prescribed dietary supplements that support the 1-CC for the patients and investigated their serum AMH levels before and after treatment.

\section{Materials and Methods}

Fifty-five patients with infertility duration of 3-7 years and at least two ART failures have been enrolled in the protocol. Forty-nine of them fulfilled all the 1-CC protocol requirements. An antral follicle count (AFC) was carried on day 3, as well as an AMH quantification. Thirty-five of these patients accepted to have a urinary BPA dosage. The dietary supplement that supports the $1-\mathrm{CC}^{19-21}$ containing vitamins $\mathrm{B} 2,3,6,9$, and 12 , zinc bisglycinate, and $N$ acetyl cysteine was prescribed for a period of 4 months. Serum AMH levels and AFCs were measured at the end of the treatment period. The patients were responsible for the costs for the treatment; six patients who could not afford to pay for the treatment, received the supplement for free.

\section{Ethical considerations}

The protocol includes treatments proposed regularly in our centers. However, the protocol has been submitted (and accepted) to the ethics committees of the clinics involved in the trial. The study was performed with products available on the market without prescription, on the basis of our previous works. ${ }^{19-21}$
AMH was assayed using the two reliable certified tests, available on the market (Beckman and Roche), depending on the clinic. However, for each patient, the same kit was used before and after treatment.

\section{BPA assays}

Urine was collected in a $250 \mathrm{~mL}$ glass container and immediately frozen at $-25^{\circ} \mathrm{C}$, to avoid ambient air contamination. BPA levels were assayed using liquid chromatography, mass spectrometry (LC-MS/MS), in an ISO-certified laboratory (Laboratoire LTB, Narbonne, France). Five milliliters of urine was first hydrolyzed enzymatically at $60^{\circ} \mathrm{C}$ for 3 hours, in the presence of an internal standard BPA-d14. Total BPA was then extracted by Solid Phase Extraction (SPE), the extract dried under a continuous flow of nitrogen, and the dry residue solubilized with $100 \mathrm{~mL}$ of the LC mobile phase. Twentyfive microliters of the sample was injected into the chromatographic system coupled with mass spectrometry tandem (UltraPerformance Liquid ChromatographyMS/MS) in negative electrospray mode (ESI-).

\section{Results}

Bisphenol quantification in urine

The mean BPA concentration $(\mu \mathrm{g} / \mathrm{L})$ observed was 2.09 $(\mathrm{SD}=3.29$, range $0-23.5)$, with the following distribution: $0: 28.6 \%$; $0-1: 28.6 \% ; 1-5: 20 \%$; and $>5: 22.9 \%$. More than two-thirds of the 35 patients had a detectable amount of BPA in their urine. No correlation between BPA and blood AMH level has been found (Fig. 2).

\section{AMH variation pretreatment and post-treatment}

Pretreatment and post-treatment AMH levels were compared using the Wilcoxon test (nonparametric test, non-Gaussian population). Thirty-three of the 49 (67\%) had an AMH level below $1 \mathrm{ng} / \mathrm{mL}$. We did observe a post-treatment increase in serum AMH: pre 1.34 , post 1.88 (Table 1 , delta $=0.54$ ), $p=0.0001$. Ultimately, the increase was not dependent either on the starting $\mathrm{AMH}$ level (probability $>\mathrm{F}=0.67$ ) or on age in the range tested (26-42 years) (probability $>\mathrm{F}=0.89$ ). The follicular count increases not significantly from 5. 4 (SD: 1.42) to 6.6 (SD: 2.27) post-treatment

At the end of the protocol and during the following 3 months, eight patients became pregnant. Their characteristics are given in Table 2.

\section{Discussion}

Premature ovarian failure and diminished ovarian reserve (DOR) syndromes are observed with increasing 


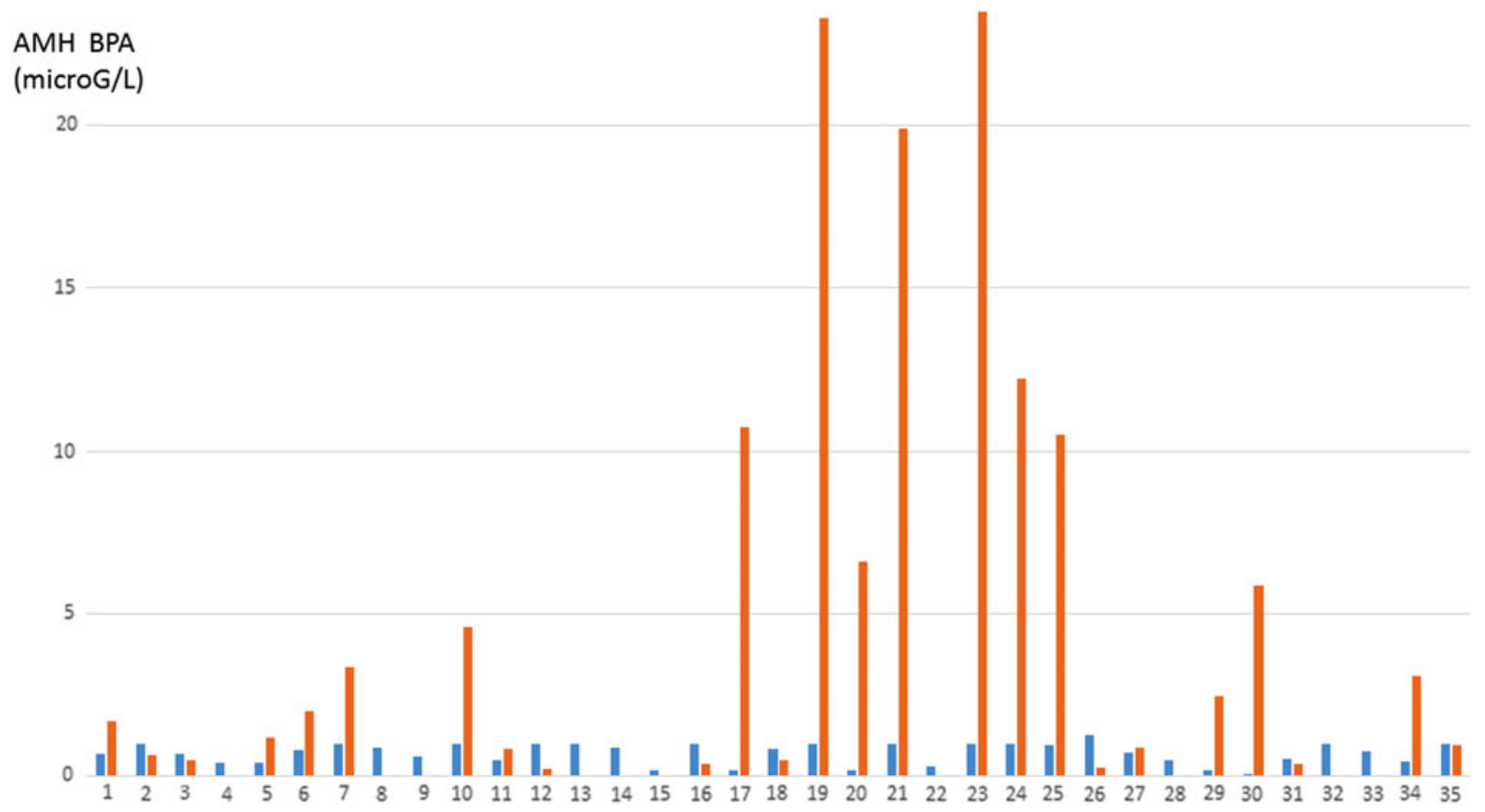

FIG. 2. Individual variations in $\mathrm{AMH}$ (blue) and urinary $\mathrm{BPA}$ ( $\mu \mathrm{g} / \mathrm{L}$, orange). $\mathrm{AMH}$, anti-Mullerian hormone; BPA, bisphenol A.

frequency in Western populations. These syndromes are mainly linked to a loss of ovarian antral follicles. In women attending a clinic for fertility treatment, higher urinary BPA concentrations were associated with lower AFCs. ${ }^{23}$ In the U.S. population, serum levels of endocrine-disrupting chemicals have been found to be associated with early menopause. ${ }^{11}$ In a female rat model, plastic-derived endocrine disruptors (BPA, DEHP, and DBP) induce severe reproductive pathologies in the pups, including, primordial follicle loss and polycystic ovarian diseases, as well as other pathologies. ${ }^{10-14}$ Our study failed to find a correlation between urinary bisphenol and the circulating $\mathrm{AMH}$ levels.

Table 1. Variations in Serum Anti-Mullerian Hormone $(\eta \mathrm{g} / \mathrm{mL})$

\begin{tabular}{cccccc}
\hline & \multirow{2}{*}{$\begin{array}{c}\text { No } \\
\text { Patients }\end{array}$} & patients & Age & & \multicolumn{3}{c}{ AMH concentration in serum } \\
\cline { 3 - 6 } & Before TT & After TT & $\boldsymbol{p}$ \\
\hline $\begin{array}{c}\text { Overall } \\
\text { (SD) }\end{array}$ & 49 & $34.9(2.5)$ & $1.34(0.98)$ & $1.88(1.2)$ & 0.0001 \\
& & & & & \\
\hline
\end{tabular}

Values pretreatment versus post-treatment (overall increase $+40 \%$ ). $\mathrm{AMH}$, Anti-Mullerian hormone; SD, standard deviation; $\Pi$, treatment.
However, it is obvious that BPA is not the only EDC; phthalates have been proposed as replacements for BPA, but they have similar noxious effects ${ }^{10-14}$; parabens are present in products for "domestic use" and even in cosmetics. Supplements that support the 1CC increase circulating AMH levels and thus to some extent may restore ovarian reserve and subsequent oocyte capacity. This confirms observations made in the mouse model. ${ }^{17}$ The most surprising feature is that increase in circulating $\mathrm{AMH}$ is independent of initial

Table 2. Characteristics of the Pregnant Patients Post-Treatment

\begin{tabular}{lcclll}
\hline & & & \multicolumn{2}{c}{$\begin{array}{c}\text { AMH level } \\
(\mu \mathbf{g} / \mathbf{L})\end{array}$} \\
\cline { 4 - 5 } & & & & & \\
& Age & BPA $(\mu \mathbf{g} / \mathrm{L})$ (urine) & Pre & Post \\
\hline NH & 34 & 0.49 & 0.7 & 0.6 \\
SA & 38 & 1.18 & 0.4 & 1.2 \\
BL & 38 & 0.84 & 0.5 & 1.9 \\
VC & 32 & 0.0 & 1 & 3.9 \\
HG & 31 & 0.0 & 0.2 & 0.5 \\
BL & 26 & NA & 1.5 & 1.6 \\
BLD & 34 & NA & 0.6 & 0.6 \\
BT & 42 & NA & 1.4 & 1.9 \\
\hline
\end{tabular}

BPA, bisphenol A; NA, not available. 
$\mathrm{AMH}$ starting levels and patient age in the range covered in this study. Another significant feature is the achievement of spontaneous ongoing pregnancies after treatment, even in patients with residual low critical AMH levels $(0.2-0.7 \eta \mathrm{g} / \mathrm{mL})$. This suggests that oocyte donation is not necessarily the only option in patients in the age range 35-42 years who have critically low AMH profiles, even post 1-CC treatment. Similar results were observed in the sperm nucleus decondensation index (SDI) of male patients who received comparable treatment. ${ }^{19-20}$ Although a global decrease in SDI was observed, it remained sometimes elevated above the normal range in some patients whose partners nonetheless achieved ongoing pregnancies, either spontaneously or post-ART treatment. We suggest that gamete quality is improved by the treatment and this is related to a decrease in general background oxidative stress and maintenance of correct/ appropriate methylation processes. Moreover, it is of common knowledge that the first stages of preimplantation development are maternally driven, through the storage of proteins and messengers RNAs accumulated during oocyte maturation. The quality of the storage decreases with maternal age and environmental stresses. Human oocytes express cytoprotective sulfonylurea receptor A (SURA) and adenosine triphosphate-sensitive $\mathrm{K}+$ (KATP) channels. ${ }^{24} \mathrm{KATP}$ channels prevent, to a certain extent, the oocyte spontaneous rise of $\mathrm{Ca}^{++}$observed in vitro. ${ }^{25}$ Intake of vitamin B3 (Nicotinamide) regulates the levels of SURA/KATP channels, ${ }^{26}$ as well as changes in NAD+/NADH ratio and ATP levels ${ }^{27,28}$ in the cardiac muscle. The heart and the oocyte have some similar biochemical features, especially if we consider the homocysteine toxicity. ${ }^{29,30}$ The treatment proposed in this study could be considered a reinforcement of the oocyte resistance to stress.

With respect to oxidative stress, our data are in accordance to some degree with the observations of Ben-Meir et al. ${ }^{31}$ : CoQ10 supplementation restores mitochondrial function during oocyte aging. Methylation is required in the last step of the CoQ10 pathway (see Fig. 1: 4-Hydroxy5-polyprenylbenzoate $+\mathrm{Me} \rightarrow$ Ubiquinone), and it is possible that CoQ10 may partly reverse premature ovarian aging related to endocrine disruptors. In conclusion, supplements that support the 1-CC should be proposed to couples who are trying to conceive, whether or not this is before an ART program. This is the first time that any treatment strategy aimed toward increasing AMH levels has been proposed. Oocyte donation is not the inevitable path for patients with DOR associated with low serum AMH, even for those in their late thirties. This is especially relevant if we consider, on the other side, the potential long-term risk of breast cancer following ovarian stimulation in young egg donors ${ }^{32}$ Evaluation of urinary EDCs (phthalates) could also be a prerequisite for this special population, despite the fact that we were not able to find a direct link between urinary BPA and low serum AMH.

\section{Acknowledgments}

We thank Mr. Olivier Brack (Statistique Industrielle $\mathrm{Khi}^{2}$ Consulting-KSCI, France) for the statistical analysis and Dr. Kay Elder (MD, PhD, Bourn Hall Clinic, Cambridge, United Kingdom), who helped us in the translation of the text. The study did not receive any financial support.

\section{Authors' Contributions}

E.S. (MD, Gyn Obst) and Y.M. (PhD, Dr Sci) designed the study, managed the study program, collected data of the full study, and wrote the article; E.S. and M.C. (MD, Gyn Obst) collected data of patients, recruited the serum samples for $\mathrm{AMH}$ and urine for BPA from patients in Lyon, and measured their AMH levels; D.C. (MD, Gyn Obst), L.J.-F. (MD, Endocrinol), and P.C. (Dr Pharm) collected data of patients, recruited the serum samples for AMH and urine for BPA from patients in Paris, and measured their AMH levels; J.C. (Dr Pharm) and M.S. (MD, Gyn Obst) collected data of patients, recruited the serum samples for $\mathrm{AMH}$ and urine for BPA from patients in Grenoble, and measured their AMH levels; and T.B. (Dr Pharm) performed the dosage of urinary BPA levels in all urine samples of the study.

\section{Author Disclosure Statement}

No competing financial interests exist.

\section{References}

1. Mak W, Weaver JR, Bartolomei MS. Is ART changing the epigenetic landscape of imprinting? Anim Reprod. 2010;7:168-176.

2. Cedar H, Bergman Y. Programming of DNA methylation patterns. Annu Rev Biochem. 2012;81:97-117.

3. Marques $C J$, Carvalho F, Sousa M, et al. Genomic imprinting in disruptive spermatogenesis. Lancet. 2004;363:1700-1702.

4. Kobayashi $H$, Sato A, Otsu E, et al. Aberrant DNA methylation of imprinted loci in sperm from oligospermic patients. Hum Mol Genet. 2007;16:25422551.

5. Aston $\mathrm{KI}$, Uren PJ, Jenkins TG, et al. Aberrant sperm DNA methylation predicts male fertility status and embryo quality. Fertil Steril. 2015;104:1388-1397.

6. Ménézo, $Y$, Khatchadourian $C$, Gharib A, et al. Regulation of S-adenosyl methionine synthesis in the mouse embryo. Life Sci 1989;44:16011609. 
7. Boxmeer JC, Steegers-Theunissen RP, Lindemans J, et al. Homocysteine metabolism in the pre-ovulatory follicle during ovarian stimulation. Hum Reprod. 2008;23:2570-2576.

8. Sato A, Otsu E, Negishi $H$, et al. Aberrant DNA methylation of imprinted loci in superovulated oocytes. Hum. Reprod. 2007;22:26-35.

9. Menezo Y, Silvestris E, Dale B, et al. Oxidative stress and alterations in DNA methylation: two sides of the same coin in reproduction. Reprod Biomed Online. 2016;33:668-683.

10. Manikkam M, Tracey $R$, Guerrero-Bosagna $C$, et al. Plastics derived endocrine disruptors (BPA, DEHP and DBP) induce epigenetic transgenerational inheritance of obesity, reproductive disease and sperm epimutations. PLoS One. 2013;8:e55387.

11. Grindler NM, Allsworth JE, Macones GA, et al. Persistent organic pollutants and early menopause in U.S. Women. PLoS One. 2015;10:e0116057.

12. Smith KW, Souter I, Dimitriadis I, et al. Urinary paraben concentrations and ovarian aging among women from a fertility center. Environ Health Perspect. 2013;121:1299-1305.

13. Bloom MS, Whitcomb BW, Chen Z, et al. Associations between urinary phthalate concentrations and semen quality parameters in a general population. Hum Reprod. 2015;30:2645-2657.

14. Nilsson E, Larsen G, Manikkam M, et al. Environmentally induced epigenetic transgenerational inheritance of ovarian disease. PLoS One. 2012;7:e36129.

15. Cooney CA, Dave AA, Wolff GL. Maternal methyl supplements in mice affect epigenetic variation and DNA methylation of offspring. J Nutr. 2002;132(8 Suppl):2393S-2400S.

16. Wolff GL, Kodell RL, Moore SR, et al. Maternal epigenetics and methyl supplements affect agouti gene expression in Avy/a mice. FASEB J. 1998;12:949-957.

17. Dolinoy DC, Huang D, Jirtle R. Maternal nutrient supplementation counteracts bisphenol A-induced DNA hypomethylation in early development. Proc Natl Acad Sci U S A. 2007;104:13056-13061.

18. Premkumar VG, Yuvaraj $S$, Shanthi $P$, et al. Co-enzyme Q10, riboflavin and niacin supplementation on alteration of DNA repair enzyme and DNA methylation in breast cancer patients undergoing tamoxifen therapy. Br J Nutr. 2008;100:1179-1182.

19. Dattilo $M$, Cornet $D, A m a r E$, et al. The importance of the one carbon cycle nutritional support in human male fertility: a preliminary clinical report. Reprod Biol Endocrinol. 2014;12:71.

20. Dattilo M, D'Amato G, Caroppo E, et al. Improvement of gamete quality by stimulating and feeding the endogenous antioxidant system: mechanisms, clinical results, insights on gene-environment interactions and the role of diet. J Assist Reprod Genet. 2016;33:1633-1648.

21. Cornet D, Amar E, Cohen M, et al. Clinical Evidence for the importance of 1-Carbon cycle support in subfertile couples. Austin J Reprod Med Infertil. 2015;2:1011.

22. Kedem A, Haas J, Geva LL, et al. Ongoing pregnancy rates in women with low and extremely low AMH levels. A multivariate analysis of 769 cycles. PLoS One. 2013;8:e81629.

23. Souter I, Smith KW, Dimitriadis I, et al. The association of bisphenol-A urinary concentrations with antral follicle counts and other measures of ovarian reserve in women undergoing infertility treatments. Reprod Toxicol. 2013;42:224-231

24. Du Q, Jovanovic S, Sukhodub A, et al. Human oocytes express ATPsensitive $\mathrm{K}(+)$ channels. Hum Reprod. 2010;25:2774-2782.

25. Fernandes G, Dasai N, Kozlova N, et al. A spontaneous increase in intracellular $\mathrm{Ca} 2+$ in metaphase II human oocytes in vitro can be prevented by drugs targeting ATP-sensitive K+ channels. Hum Reprod. 2016;31: 287-297.

26. Sukhodub A, Sudhir R, Du Q, et al. Nicotinamide-rich diet improves physical endurance by up-regulating SUR2A in the heart. J Cell Mol Med. 2011;15:1703-1712.

27. Mohamed Abdul KS, Jovanović S, Du Q, et al. Mild hypoxia in vivo regulates cardioprotective SUR2A: a role for Akt and LDH. Biochim Biophys Acta. 2015;1852:709-719.

28. Mohamed Abdul KS, Jovanović S, Du Q, et al. A link between ATP and SUR2A: a novel mechanism explaining cardioprotection at high altitude. Int J Cardiol. 2015;189:73-76.

29. Hoffman M. Hypothesis: hyper-homocysteinemia is an indicator of oxidant stress Med Hypotheses. 2011;77:1088-1093.
30. Ménézo Y, Lichtblau I, Elder K. New insights into human pre-implantation metabolism in vivo and in vitro. J Assist Reprod Genet. 2013; 30:293303.

31. Ben-Meir A, Burstein E, Borrego-Alvarez A, et al. Coenzyme Q10 restores oocyte mitochondrial function and fertility during reproductive aging. Aging Cell. 2015;14:887-895.

32. Schneider J, Lahl J, Kramer W. Long term breast cancer risks following ovarian stimulation in young egg donors: a call for follow-up, research and informed consent. Reprod. Biomed Online. 2017;34:480-485.

Cite this article as: Silvestris $E$, Cohen $M$, Cornet $D$, JacquessonFournols L, Clement $P$, Chouteau J, Schneider M, Besnard T, Ménézo $Y$ (2017) Supporting the one-carbon cycle restores ovarian reserve in subfertile women: absence of correlation with urinary bisphenol A concentration, BioResearch Open Access 6:1, 104-109, DOI: 10.1089/ biores.2017.0016.

Abbreviations Used
$1-C C=$ one-carbon cycle
$\mathrm{AFC}=$ antral follicle count
$\mathrm{AMH}=$ anti-Mullerian hormone
$\mathrm{BPA}=$ bisphenol $\mathrm{A}$
$\mathrm{DBP}=$ dibutyl phthalate
$\mathrm{DEHP}=$ di(2-ethylhexyl)phthalate
$\mathrm{DOR}=$ diminished ovarian reserve
$\mathrm{EDC}=$ endocrine disrupting chemicals
$\mathrm{SDI}=$ sperm nucleus decondensation index

